\title{
Convection Heat Transfer in a Horizontal Annulus with an Oscillating Elastic Outer Boundary
}

\author{
Nikolai Kozlov \\ Institute of Continuous Media Mechanics UrB RAS \\ 1, Ac. Koroleva St., Perm, Russia \\ kozlov.n@icmm.ru
}

\section{Extended Abstract}

Heat and mass transfer is crucial for many technological processes, and methods of its intensification are being actively developed nowadays. One approach to this consists in applying vibrations that can have noticeable effect on systems possessing an interface or a density inhomogeneity.

In the presented work, convection and heat transfer are studied experimentally in a horizontal coaxial layer whose outer wall is formed by an elastic silicone tube of the radius $R_{2}$ and the inner one by a cylindrical copper heat exchanger $\left(R_{1}\right)$. An aqueous solution of glycerine with a concentration of 0.90 is used as the working fluid. The temperature of the outer wall $T_{2}$ is kept constant by pumping a coolant through a thermostatic jacket, inside which the experimental model is placed. The temperature of the heat exchanger $T_{1}$ is set using an electric heater placed inside it ( $T_{1}>T_{2}$ ). The elastic wall is driven by two linear motors, installed symmetrically to the left and to the right of the container and performing synchronous vibrations with the radian frequency $\Omega$ and the amplitude $b$. As a result, the elastic boundary is periodically contracted and extended along the horizontal axis, its time-average shape remaining circular. A thermocouple is used for measuring $\Theta=T_{1}-T_{2}$, and thermistors for $T_{2}$. The fluid flow is studied using particle image velocimetry (PIV).

In the absence of vibrations, $\Theta=\Theta_{0}$ and the natural convection is observed in the form of two symmetric rolls [1]. Vibrations lead to the generation of steady time-average flows in the form of eight pairs of rolls regularly distributed along the azimuth. The flows are two-dimensional, i.e. invariant along the cylinder axis with an exception of the regions near the flanges. Vibrational convection enhances heat transfer: $\Theta$ decreases. The natural convection intensity is determined by the Rayleigh number $\mathrm{Ra} \equiv g \beta \Theta\left(R_{2}-R_{1}\right)^{3} /(v \chi)$. The vibrational convection intensity depends on the pulsation Reynolds number $\operatorname{Re}_{p} \equiv \Omega b^{2} / v$, and the steady-flow structure - on the dimensionless

frequency $\omega \equiv \Omega\left(R_{2}-R_{1}\right)^{2} / v$. If $\omega=$ const, the heat transfer rate (the Nusselt number $\mathrm{Nu}$ ) and the flow structure depend on the ratio between $\mathrm{Ra}$ and $\mathrm{Re}_{p}$. The analysis of the dimensionless parameters shows that the thermal vibrational mechanism [2] is not important in the present experiments, but the steady streaming is. In the range $\mathrm{Ra}<10^{5}, 30<\omega<80$ and $\operatorname{Re}_{p}>4.6 \cdot 10^{-3} \mathrm{Ra}^{2 / 3}, \mathrm{Nu}$ is determined by $\mathrm{Re}_{p}$ and weakly depends on $\mathrm{Ra}$, the scaling $\mathrm{Nu} \sim \operatorname{Re}_{p}^{0.3}$ is observed.

The obtained experimental results prove the steady streaming to be an efficient mechanism for the heat transfer intensification. In the experiments, it was possible to achieve a pronounced increase in the heat transfer rate. In particular, at $\mathrm{Ra}=1.13 \cdot 10^{3}, \omega=31.3$ and $\operatorname{Re}_{p}=10.4$, the maximum heat transfer enhancement $\Theta_{0} / \Theta=2.18$ was observed that corresponds to a $118 \%$ increase in $\mathrm{Nu}$.

\section{Acknowledgements}

The research was supported by the Russian Science Foundation (project 17-71-10189). 


\section{References}

[1] T. H. Kuehn, R. J. Goldstein, "An experimental and theoretical study of natural convection in the annulus between horizontal concentric cylinders," J. Fluid Mech., vol. 74, no. 4, pp. 695-719, 1976.

[2] G. Z. Gershuni and D. V. Lyubimov, Thermal Vibrational Convection. New York, USA: Wiley, 1998. 